\title{
Kernel nullers for an arbitrary number of apertures
}

\author{
Romain Laugier, Nick Cvetojevic, and Frantz Martinache
}

\author{
Université Côte d'Azur, Observatoire de la Côte d'Azur, CNRS, Laboratoire Lagrange, France \\ e-mail: romain.laugier@oca.eu
}

Received 8 July 2020 / Accepted 18 August 2020

\begin{abstract}
Context. The use of interferometric nulling for the direct detection of extrasolar planets is in part limited by the extreme sensitivity of the instrumental response to tiny optical path differences between apertures. The recently proposed kernel-nuller architecture attempts to alleviate this effect with an all-in-one combiner design that enables the production of observables inherently robust to residual optical path differences $(\ll \lambda)$.

Aims. To date, a unique kernel-nuller design has been proposed ad hoc for a four-beam combiner. We examine the properties of this original design and generalize them for an arbitrary number of apertures.

Methods. We introduce a convenient graphical representation of the complex combiner matrices that model the kernel nuller and highlight the symmetry properties that enable the formation of kernel nulls. The analytical description of the nulled outputs we provide demonstrates the properties of a kernel nuller.

Results. Our description helps outline a systematic way to build a kernel nuller for an arbitrary number of apertures. The designs for three- and six-input combiners are presented along with the original four-input concept. The combiner grows in complexity with the square of the number of apertures. While one can mitigate this complexity by multiplexing nullers working independently over a smaller number of sub-apertures, an all-in-one kernel nuller recombining a large number of apertures appears as the most efficient way to characterize a high-contrast complex astrophysical scene.

Conclusions. Kernel nullers can be designed for an arbitrary number of apertures that produce observable quantities robust to residual perturbations. The designs we recommend are lossless and take full advantage of all the available interferometric baselines. They are complete, result in as many kernel nulls as the theoretically expected number of closure-phases, and are optimized to require the smallest possible number of outputs.
\end{abstract}

Key words. instrumentation: interferometers - techniques: high angular resolution - techniques: interferometric planets and satellites: detection

\section{Introduction}

The last 25 years have seen the detection of more than 4000 exoplanets (Schneider et al. 2011). Despite the indirect nature of most detections, existing observations already provide us with a wealth of information on the properties of exoplanetary systems: their mass, size, and orbital elements. Even so, direct detection of a planet's reflected or radiated light, which would permit the direct spectral analysis of a large sample of targets, remains an exciting prospect that will contribute to further characterizing individual planets, in particular the properties of their atmospheres (Marois et al. 2008; Zurlo et al. 2016).

The use of coronagraphic instruments is now leading to the detection of young giant planets in wide orbits around nearby stars (Macintosh et al. 2015; Chauvin et al. 2017; Mesa et al. 2019). This success is advanced by the continued improvements of extreme adaptive optics systems (Sauvage et al. 2016; Lozi et al. 2018; Boccaletti et al. 2020). For smaller separations approaching the diffraction limit (below $\sim 3 \lambda / D$ ), small residual wavefront errors still dominate the error budget and coronagraphic solutions become less favorable.

Lacour et al. (2019) have demonstrated the advantages brought by long-baseline interferometry for the characterization of extrasolar planets. This observing mode takes advantage of the spatial filtering provided by the resolving power of each of the $8 \mathrm{~m}$ telescopes of the Very Large Telescope Interferometer (VLTI), coupled into single mode fibers to reach the required contrast. Interferometric nullers (Bracewell 1978; Colavita et al.
2009; Serabyn et al. 2019; Hoffmann et al. 2014; Defrère et al. 2015; Norris et al. 2020) offer the possibility to explore smaller angular separations through the use of fragmented apertures and long-baseline interferometry. Some combining solutions have been found that optimize the rejection of resolved stars (Angel \& Woolf 1997; Guyon et al. 2013). The exploitation of these instruments is still limited by their vulnerability to optical path difference (OPD) errors, and requires sophisticated statistical analysis, like those proposed by Hanot et al. (2011) and Defrère et al. (2016), and more recently used by Norris et al. (2020) to disentangle the off-axis astrophysical signal from the effects of unwanted OPDs.

Classical long-baseline and Fizeau interferometry make extensive use of the production of robust observables, like closure phases (Jennison 1958), and their generalized form, kernel phases (Martinache 2010), to sidestep the limitations brought by the OPD residuals. This approach has provided reliable performance at very small separations, down to one resolution element and below.

Bringing together the robustness of interferometric observables and the photon-noise suppression of nulling is an exciting perspective as it opens a novel high-contrast, high-precision regime. The double-Bracewell architecture (Angel \& Woolf 1997) was noted to offer such robustness (Velusamy et al. 2003) when implemented with the adequate phase shift between the two stages (known as sin-chop). A different approach was later proposed by Lacour et al. (2014), which exploits the measurement of fringes in the leakage light. 
Martinache \& Ireland (2018) introduced an alternative, more efficient solution for a four-telescope beam-combiner architecture that produces six nulled outputs. By analyzing the response of these outputs to parasitic OPDs (instrumentally or atmospherically induced phase error), the authors identify linear combinations of outputs that are robust to these aberrations to second order. The solution they propose uses a four-input nuller that provides three nulled kernel observables.

In this paper we look for the properties that ensure that a combiner will produce kernel nulls. They help outline a general strategy for the design of kernel nullers for an arbitrary number of apertures.

\section{Analysis of the existing four-input kernel-nulling architecture}

\subsection{Kernel-nulling approach}

Using the nuller architecture laid out by Martinache \& Ireland (2018) for a four-beam interferometer as the starting point and reexamining its properties, we look into ways of generalizing this special case to a wider range of configurations involving different numbers of apertures.

The inner structure of a homodyne interferometric combiner (nulling or not) is conveniently represented by a combiner matrix $\mathbf{M}$ that acts on a vector $\mathbf{z}$ of input electric fields and leads to the production of an output electric field vector $\mathbf{x}$ :

$\mathbf{x}=\mathbf{M} \cdot \mathbf{z}$.

A detector then records the intensity associated with the square norm of this output electric field.

The fact that only the square norm of the field is recorded has two consequences. The first is that the response of the combiner is insensitive to the absolute phase of the input electric field: one of the sub-apertures can therefore be arbitrarily picked as a reference, and the phases of the different electric fields sampled by the other sub-apertures are measured relative to that reference. The second is that the output intensity is equally insensitive to any global phase shift $\phi$ applied to any row $\mathbf{m}$ of the matrix $\mathbf{M}$ describing the combiner. This is of consequence when identifying distinct combinations (or rows).

Assuming that the recombiner is fed by a balanced array of identical sub-apertures, the complex amplitude of the input electric field can be described by a vector of phasors. We further assume that the combiner benefits from a fringe tracker that, although not perfect, brings the system close to its nominal state. The fringe tracking residuals are assumed to be small and all phasors $e^{-j \varphi_{k}}$ are approximated using the following expansion, where $j$ is the imaginary unit:

$z_{k}=e^{-j \varphi_{k}} \approx 1-j \varphi_{k}$

Since only intensities are measured, the overall response of the system is a quadratic function of the perturbation phase vector. Martinache \& Ireland (2018) thus use this description to look at the properties of the second-order derivative of the intensity relative to the phase. One of the $n_{a}$ sub-apertures being used as a phase reference, there are $n_{a}-1$ degrees of freedom, and $n_{d}=n_{a} *\left(n_{a}-1\right) / 2$ such derivatives. This response can be stored in a $n_{o} \times n_{d}$ matrix $\mathbf{A}$ called the matrix of second-order derivatives, where $n_{o}$ is the number of relevant outputs. Linear combinations of rows of $\mathbf{A}$ that equal 0 cancel out the secondorder intensity deviations caused by small input phase errors. The same linear combination applied to the intensity measured after the recombiner will be equally insensitive to small input phase errors. We refer to these linear combinations as kernel outputs or kernel nulls when applied to a collection of nulled outputs.

The rank of $\mathbf{A}$ and the possibility of forming such robust observables rely entirely on the properties of the matrix $\mathbf{M}$, and therefore does not depend on the geometry of the input array. However, the question of whether a kernel null carries astrophysically relevant information also depends on the configuration of the array. Throughout this work, phase and amplitude contributions are considered independently, but their coupled contribution is neglected. In Sects. 2.2, 2.3 and 3, of this paper we introduce a convenient visual representation of the structure of $\mathbf{M}$, and further examine the properties of the combiner.

\subsection{Visualization of complex combiner matrices}

The effect of the matrix $\mathbf{M}$ on the complex amplitude of the input electric field can be conveniently visualized by a series of plots of the complex plane. For a given combiner, each input is represented by a colored arrow that, in the absence of environmental perturbation, is aligned with the real axis. Each plot illustrates the effect of a row of $\mathbf{M}$ on such inputs: the resulting electric field is the sum of all colored arrows present in the plot. A nuller is characterized by several outputs for which the sum of the arrows, associated with the electric fields, sum up to zero. These complex matrix plots (CMPs) are used throughout this work to describe several nuller designs of varying complexity.

\subsection{From real to complex nulls}

The architecture of the kernel nuller described in Martinache \& Ireland (2018) builds from an initial all-in-one four-beam nuller whose overall effect can be described by the following matrix:

$\mathbf{N}_{4}=\frac{1}{\sqrt{4}}\left[\begin{array}{cccc}1 & 1 & 1 & 1 \\ 1 & 1 & -1 & -1 \\ 1 & -1 & 1 & -1 \\ 1 & -1 & -1 & 1\end{array}\right]$.

This matrix is real. Each nulled row of $\mathbf{N}_{\mathbf{4}}$ recombines distinct arrangements of the four input electric fields such that the coefficients on the corresponding rows sum up to zero, as represented in Fig. 1, with arrows aligned with the real axis: two positive (or not phase-shifted), and two negative (or phase-shifted by $\pi$ ). As discussed in this reference, this nuller does not allow the formation of kernels because the output intensities it produces are a degenerate function of the target information and input phase perturbations. The outputs of this nuller can however be fed to a second stage, described by the following matrix:

$\mathbf{S}_{4}=\frac{1}{\sqrt{4}}\left[\begin{array}{cccc}2 & 0 & 0 & 0 \\ 0 & 1 & e^{j \frac{\pi}{2}} & 0 \\ 0 & e^{j \frac{\pi}{2}} & 1 & 0 \\ 0 & 1 & 0 & e^{j \frac{\pi}{2}} \\ 0 & e^{j \frac{\pi}{2}} & 0 & 1 \\ 0 & 0 & 1 & e^{j \frac{\pi}{2}} \\ 0 & 0 & e^{j \frac{\pi}{2}} & 1\end{array}\right]$,

which leaves the bright output untouched, but further splits the nulled ones and selectively introduces $\pi / 2$ phase shifts. The overall effect of the combiner is described by a now complex combiner matrix, result of the product $\mathbf{M}_{4}=\mathbf{S}_{4} \cdot \mathbf{N}_{4}$. The CMPs of this modified combiner, shown in Fig. 2, offer a more easily readable description of its effect, with components of the output electric field no longer simply aligned with the real axis, but spanning the complex plane.

The new complex configuration enables the greater diversity that is required to disentangle the otherwise degenerate 

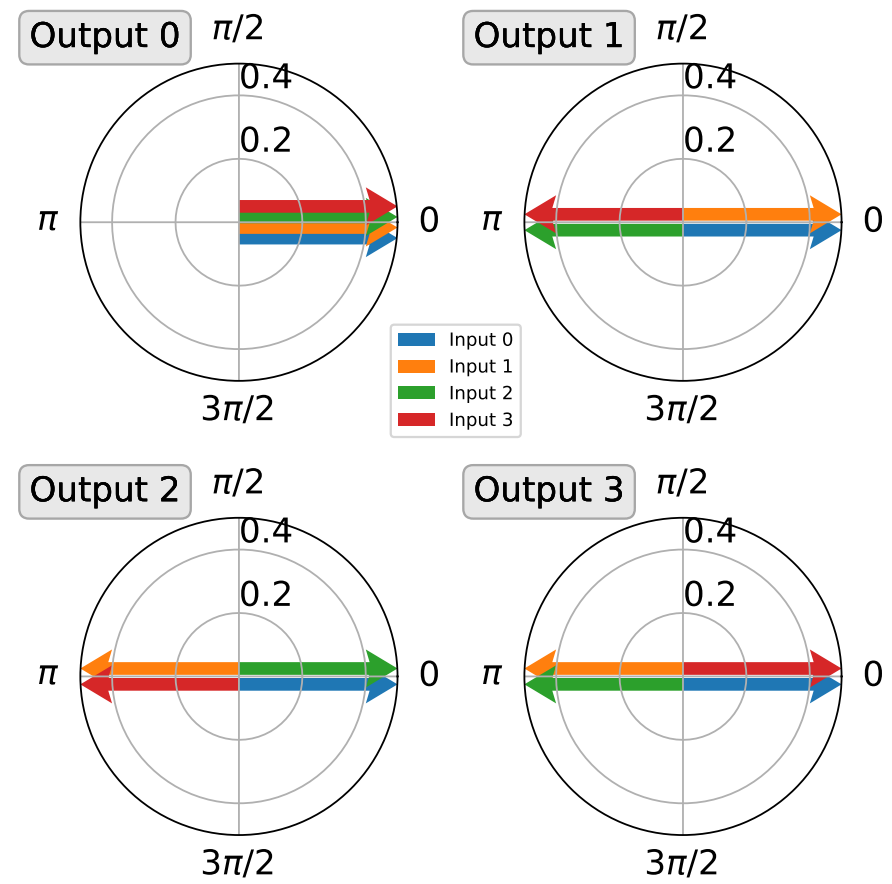

Fig. 1. CMP of the matrix $\mathbf{N}_{4}$ in Eq. (3), representing a four-input nulling combiner. The first row constitutes the bright channel, with all inputs combined constructively. Each output is a contribution of all the inputs, and not just a pair of them, which prevents the direct interpretation through the uv plane.

effect that environmental perturbations have on the input electric fields. The modified nuller indeed features more outputs than inputs, and a close examination of the CMPs in Fig. 2 shows that all six combinations offer a distinct arrangement of the four input fields. The construction of a larger number of distinct nulls is one of the requirements for the existence of a non-empty left null space for A described in Sect. 2.1. Pairs of outputs produce the same response to environmental effects while still producing different response to off-axis light.

\section{Properties of conjugate pairs of nulls}

\subsection{Kernel outputs}

Identifying the kernel-forming combinations of outputs no longer requires building the second-order derivative matrix $\mathbf{A}$, but can be achieved by examination of the CMP representation of the nuller. Figure 2 lays out, side by side, the two outputs leading to one kernel-null observable. Since close examination of such pairs of outputs reveals that the electric field combination patterns are the mirror image of one another, we call them enantiomorph.

Given that the measured intensity associated with any output is insensitive to a global phase shift, such a shift can always be applied so as to align the arrow corresponding to the phase reference input with the real axis, and point it towards the positive direction. After such a rotation is applied, enantiomorph outputs simply become complex conjugate. This makes it possible to write simple equations that describe the two key properties of kernel nulls: their robustness to small phase perturbation, and the antisymmetric nature of the signal they provide.

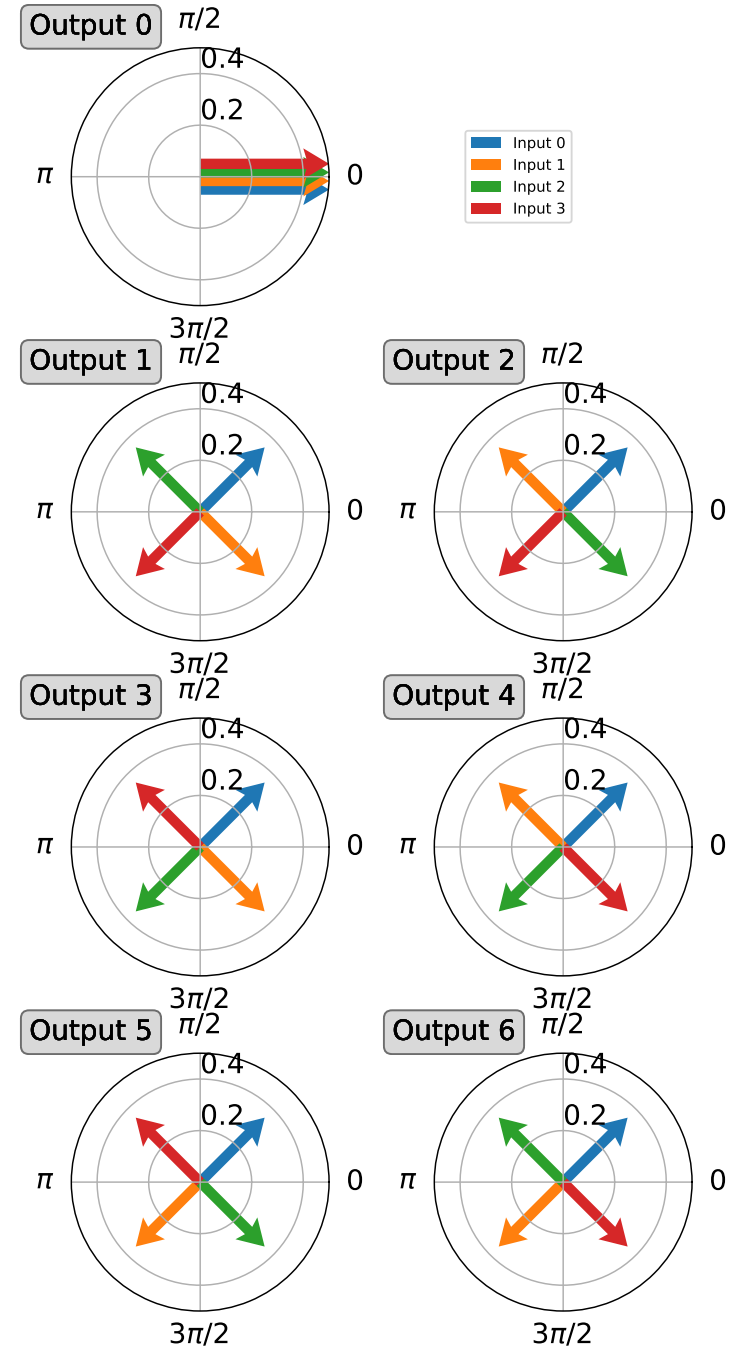

Fig. 2. CMP of the $\mathbf{S}_{4} \cdot \mathbf{N}_{4}$ combination. The first output is the bright channel for which all the inputs add up constructively. The vectors are staggered for readability. Pairs of nulled rows represented side by side are mirror images of each other (enantiomorph). The amplitude of the phasors is reduced compared to Fig. 1 due to additional splitting.

\subsection{Robustness}

We consider $\mathbf{m}_{1}$ and $\mathbf{m}_{2}$ a conjugate pair of null rows of $\mathbf{M}$ :

$\mathbf{m}_{2}=\mathbf{m}_{1}^{*}$.

A corresponding kernel null $\kappa(\mathbf{z})$ writes as the difference of the two measured intensities:

$\kappa(\mathbf{z})=\left|\mathbf{m}_{1} \mathbf{z}\right|^{2}-\left|\mathbf{m}_{2} \mathbf{z}\right|^{2}=\mathbf{m}_{1} \mathbf{z}\left(\mathbf{m}_{1} \mathbf{z}\right)^{*}-\mathbf{m}_{2} \mathbf{z}\left(\mathbf{m}_{2} \mathbf{z}\right)^{*}$.

Using (5) and (6) gives

$\kappa(\mathbf{z})=\mathbf{m}_{1} \mathbf{z}\left(\mathbf{m}_{1} \mathbf{z}\right)^{*}-\mathbf{m}_{1}^{*} \mathbf{z}\left(\mathbf{m}_{1}^{*} \mathbf{z}\right)^{*}$.

In the case of the approximation mentioned in Eq. (2), we have

$$
\begin{aligned}
\kappa(\mathbf{z})= & \mathbf{m}_{1}(\mathbf{a}+j \boldsymbol{\varphi})\left(\mathbf{m}_{1}(\mathbf{a}+j \boldsymbol{\varphi})\right)^{*} \\
& -\mathbf{m}_{1}^{*}(\mathbf{a}+j \boldsymbol{\varphi})\left(\mathbf{m}_{1}^{*}(\mathbf{a}+j \boldsymbol{\varphi})\right)^{*},
\end{aligned}
$$

where $\mathbf{a}$ is a vector of ones. Developing this expression, since $\mathbf{m}_{1} \mathbf{a}=0$ and $\mathbf{m}_{1}^{*} \mathbf{a}=0$, the only terms left are those containing only the imaginary perturbation term $j \varphi$ :

$\kappa(\mathbf{z})=\mathbf{m}_{1} j \varphi\left(\mathbf{m}_{1} j \varphi\right)^{*}-\mathbf{m}_{1}^{*} j \varphi\left(\mathbf{m}_{1}^{*} j \varphi\right)^{*}$. 
Distributing the conjugate operator gives

$\kappa(\mathbf{z})=-\mathbf{m}_{1} j \varphi \mathbf{m}_{1}^{*} j \varphi+\mathbf{m}_{1}^{*} j \varphi \mathbf{m}_{1} j \varphi$,

and therefore $\kappa(\mathbf{z})=0$ due to the commutativity. This shows that the subtraction of intensity of complex conjugate pairs of nulled outputs always produces a kernel null that is robust to arbitrary imaginary phasors, to which the small input phase aberrations are approximated.

This property also applies to arbitrary purely real input electric fields that would correspond to pure photometric error generated by fluctuations of the coupling efficiencies. Considering a purely real input vector $\mathbf{a}$, we have

$\kappa(\mathbf{z})=\mathbf{m}_{1} \mathbf{a}\left(\mathbf{m}_{1} \mathbf{a}\right)^{*}-\mathbf{m}_{1}^{*} \mathbf{a}\left(\mathbf{m}_{1}^{*} \mathbf{a}\right)^{*}$.

Distributing the conjugate operator gives

$\kappa(\mathbf{z})=\mathbf{m}_{1} \mathbf{a} \mathbf{m}_{1}^{*} \mathbf{a}-\mathbf{m}_{1}^{*} \mathbf{a} \mathbf{m}_{1} \mathbf{a}$,

and therefore $\kappa(\mathbf{z})=0$ due to the commutativity.

At any instant the subtraction of the signals recorded by conjugate (or more generally enantiomorph) outputs forms a kernel null. Conjugate pairs of nulls allow the formation of kernel nulls. This property generalizes to enantiomorph pairs of nulls through the rotation by a single common phasor. A complementary approach for the identification of robust combinations of outputs is the use of the singular value decomposition (SVD) of the second-order derivative matrix $\mathbf{A}$, as mentioned by Martinache \& Ireland (2018), which ensures the identification of all the robust combinations of outputs.

This behavior can be illustrated by adding different phased contributions to the inputs, and plotting the resulting electric field on top of the original perfectly cophased CMPs (dashed lines). The first panel of Fig. 3 uses this representation of the combined light to illustrate how small input phase aberrations affect the amplitude (and therefore the intensity) of the combiner's outputs. In particular it shows how, for small phase errors, conjugate pairs of nulls suffer the same leakage-light intensity.

\subsection{Symmetry of the response}

The second and third panels of Fig. 3 show how input light coming from a significantly off-axis source (input phases $\phi \geq 1$ radian) propagates to the nulled outputs. They highlight how this off-axis light produces different intensities at the outputs of the conjugate pairs, translating into a kernel-null signal.

For a combiner that is fed by an array of apertures collecting the light from the sky, the value of this response as a function of the incidence of the light is the response map of the interferometer and depends on the position of each of the apertures. Martinache \& Ireland (2018) note how this map is antisymmetric, therefore providing a rejection of the photosphere of stars and symmetric circumstellar disks that could hide a planetary companion, and provide the astrometry of such companions without ambiguity. Using our formalism, we can demonstrate this antisymmetric property for any aperture configuration. If $\mathbf{z}$ and $\mathbf{z}^{\prime}$ are two input electric field vectors coming from sources located at symmetric positions in the field of view, then

$\mathbf{z}^{\prime}=\mathbf{z}^{*}$

Considering again a conjugate pair of null rows $\mathbf{m}_{1}$ and $\mathbf{m}_{2}$, and by substitution of (13) into (6) we get

$\kappa(\mathbf{z})=\mathbf{m}_{1} \mathbf{z}^{\prime *} \mathbf{m}_{1}^{*} \mathbf{z}^{\prime}-\mathbf{m}_{2} \mathbf{z}^{\prime *} \mathbf{m}_{2}^{*} \mathbf{z}^{\prime}$.
After substitution of (5), this becomes

$\kappa(\mathbf{z})=\mathbf{m}_{2}^{*} \mathbf{z}^{* *} \mathbf{m}_{2} \mathbf{z}^{\prime}-\mathbf{m}_{1}^{*} \mathbf{z}^{\prime *} \mathbf{m}_{1} \mathbf{z}^{\prime}$.

This leads to the conclusion that the response is antisymmetric:

$\kappa(\mathbf{z})=-\kappa\left(\mathbf{z}^{\prime}\right)$.

Conversely, we can also extract from this pair of nulls the complementary observable

$\tau(\mathbf{z})=\mathbf{m}_{1} \mathbf{z}\left(\mathbf{m}_{1} \mathbf{z}\right)^{*}+\mathbf{m}_{2} \mathbf{z}\left(\mathbf{m}_{2} \mathbf{z}\right)^{*}$

whose response is symmetric. The observables $\kappa$ and $\tau$ therefore carry complementary information on the target field, much like the amplitude and phase of complex visibility in classical interferometry.

Although $\tau$ does not have the same robustness to aberrations, there may be ways to use it with the processing methods employed by Hanot et al. (2011) and Norris et al. (2020) so as to provide additional information on the target in different science cases. The kernel null $\kappa$ is best suited for the study of high-contrast non-symmetrical features such as planetary companions, while $\tau$ may be used to study brighter symmetrical features such as debris disks or stellar envelopes. Combining the two types of observables could enable image reconstruction.

The $\tau$ observables carry some information about the input phase errors. We can use their values over the course of a scan or modulation of the OPDs to locate the setpoint of the kernel nuller, for which they will reach a minimum.

\section{Construction of new nullers}

\subsection{Blueprints of kernel-nulling matrices}

The properties used in Sects. 3.2 and 3.3 to demonstrate the robustness of kernel nullers to small phase perturbations can be used as constraints to guide the design of an arbitrary kernelnuller matrix. For the output of any row $l$ to provide an on-axis null, the matrix coefficients must satisfy

$\sum_{k=0}^{n_{a}-1} M_{k, l}=0$.

Output intensities are unchanged when the coefficients of a row are all multiplied by a common phasor. We therefore apply one such phasor so as to get $\operatorname{Arg}\left(M_{0, l}\right)=0$. We also build output zero by combining all the inputs without any phase offset: $\operatorname{Arg}\left(M_{k, 0}\right)=0$.

Simple solutions to Eq. (18) for a balanced array can be found by picking arrangements of uniformly spaced phase values in the $[0,2 \pi]$ interval as can be seen of Figs. 2, 4, and 6. The phase of each coefficient is therefore a multiple of $\Phi_{0}=2 \pi / n_{a}$. On the CMPs seen thus far, this would result in the rotation of all of the arrows on the nulled outputs until the one associated with input zero is aligned with the real axis in the positive direction. With these constraints in place, outputs will only differ in the order in which the remaining $n_{a}-1$ phase offsets are associated with the inputs. The maximum number of distinct nulled outputs is therefore

$n_{\max }=\left(n_{a}-1\right) !$.

The phase term $\phi_{k, l}$ writes

$\phi_{k, l}=c_{k, l} \Phi_{0}$, 

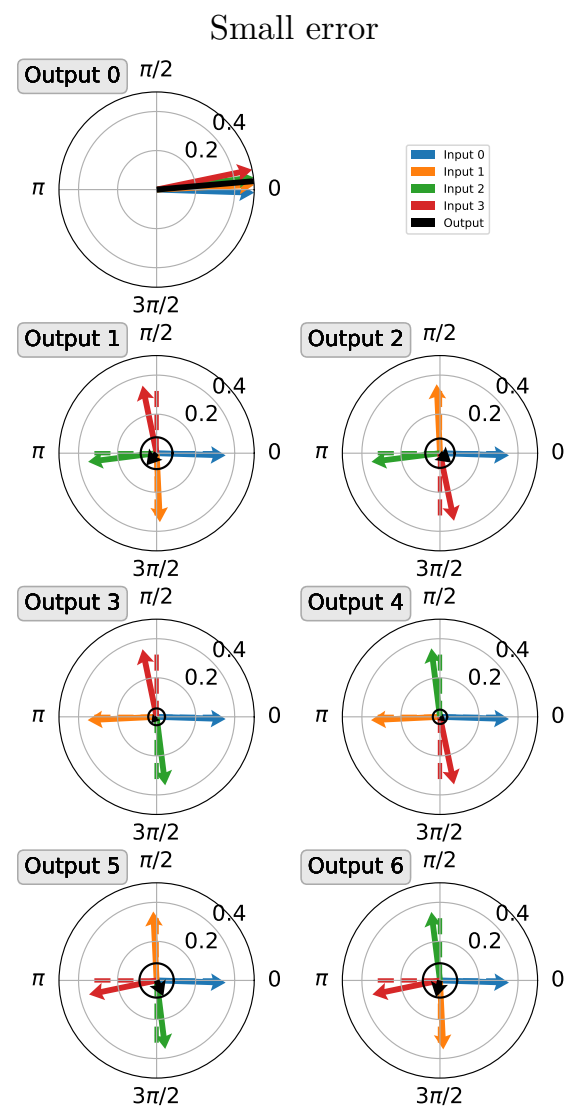

Partially resolved
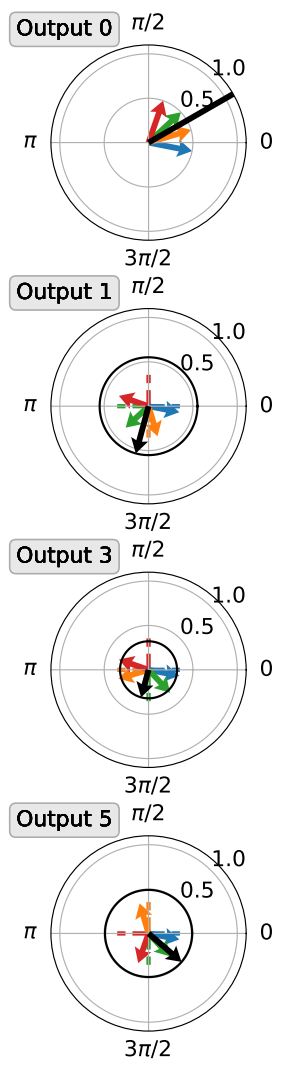
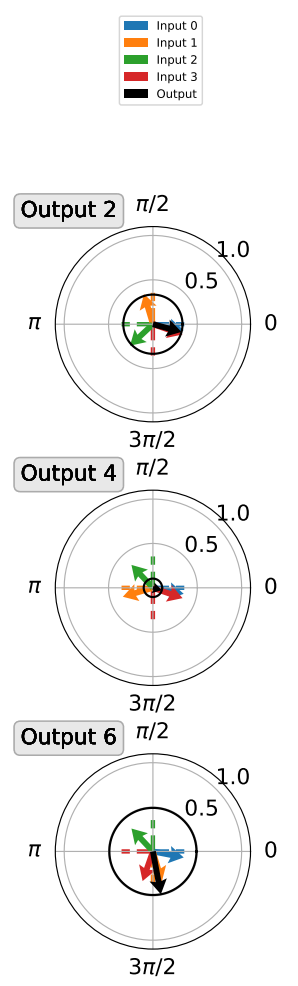
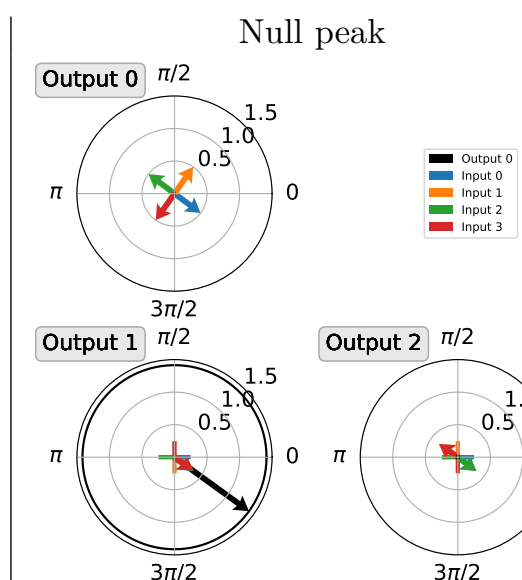

Output $2 \pi / 2$

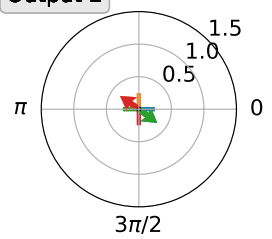

Output $3 \pi / 2$

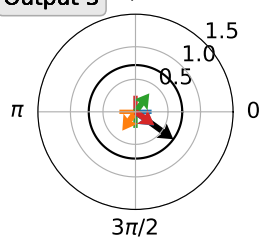

Output $4 \pi / 2$
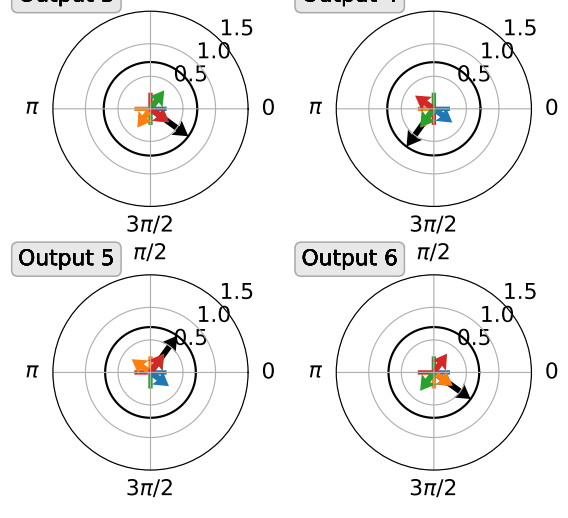

Fig. 3. CMP for a four-input combiner, representing the coefficients of the combiner matrix $\mathbf{M}_{4}^{\prime}$ (dashed lines), functionally equivalent to $\mathbf{M}_{4}$, and the contributions of the complex amplitude of an example input electric field (solid arrows) to the output electric field represented in black. Most dashed lines are hidden under the arrows. A black circle of radius equal to the modulus of this output is plotted for a visual cue, its area being proportional to the corresponding intensity. Enantiomorph pairs that generate kernel combinations by subtraction are represented side by side. As do Martinache \& Ireland (2018), we use the example of the VLTI UT configuration observing at zenith at a wavelength of 3.6 $\mu m$. Left panel: source located at 0.2 mas from the optical axis and for which the corresponding input phase shifts are within the small phase approximation. As a result, the output intensities within each pair are fully correlated and result in no kernel signal. Center panel: source located 1.1 mas off-axis, which generates larger phase shifts. As a result, the null intensities from the enantiomorph pairs begin to decorrelate and generate kernel-null signal. Right panel: source located 4.3 mas from the optical axis, in the position where the first nulled output peaks. At this position the second output gets to zero.

where $c_{k, l} a$ is the $k$ th term of the $l$ th possible combination on the circle. In general, a complex coefficient of $\mathbf{M}$ will therefore write

$$
M_{k, l}=a_{l} \cdot e^{j \phi_{k, l}},
$$

where $a_{l}$ is a real coefficient, normalizing the matrix, so that $\mathbf{M}$ represents a lossless beam-combiner for which each column vector is of unit norm. As mentioned in Appendix A, this condition on the norm is necessary (but not sufficient) to ensure that the matrix represents a lossless beam combiner, and one solution for it is to have

$$
\left\{\begin{array}{ll}
a_{l}=\frac{1}{\sqrt{n_{a}}} & \text { for the bright output } \\
a_{l}=\frac{1}{\sqrt{n_{a}}} \sqrt{\frac{n_{a}-1}{n_{\text {null }}}} & \text { for the nulled outputs }
\end{array},\right.
$$

where $n_{\text {null }}$ is the number of nulled outputs. Normalization is not mandatory to study the qualitative properties of the combiner, but it is necessary to study their throughput in a quantitative manner and their practical implementation.

The matrix M obtained with Eq. (21), represents a combiner for which pairs of complex conjugate nulls can be subtracted to build the kernel nulls that are the focus of this work.
Table 1. Growth of kernel-nuller combiners with the number of apertures.

\begin{tabular}{lccc}
\hline \hline $\begin{array}{l}\text { Inputs } \\
n_{a}\end{array}$ & $\begin{array}{c}\text { Distinct nulls } \\
n_{\max }\end{array}$ & $\begin{array}{c}\text { Indep. nulls } \\
n_{\text {indep. }}\end{array}$ & $\begin{array}{c}\text { Kernel nulls } \\
n_{\mathrm{kn}}\end{array}$ \\
\hline 3 & 2 & 2 & 1 \\
4 & 6 & 6 & 3 \\
5 & 24 & 12 & 6 \\
6 & 120 & 20 & 10 \\
7 & 720 & 30 & 15 \\
\hline
\end{tabular}

\subsection{Information redundancy}

As shown by Eq. (19) and Table 1, the number of nulled outputs that would result from a strict application of these blueprint rules rapidly grows as the factorial of the number of inputs. However, for numbers of apertures larger than four, although all the nulls produced with the presented scheme are distinct, some of them do not carry new information on the target as their response function to an off-axis signal is a linear combination of the response function of other nulls. 
Here we analyze this property empirically by examining the response maps (analogous to Figs. 5 and 7 of Martinache \& Ireland 2018) and assembling them as vectors of a set of nulled outputs and a set of kernel outputs. The ranks of these sets provide the number of independent observables produced by the combiner. Although we were not able to link this property to particular traits of the combinations, the largest number of independent kernel nulls obtainable by a given nonredundant array of apertures was always the same as the number of independent closure phases, which is in agreement with the expectations set by Martinache \& Ireland (2018). For any nonredundant array of apertures, this number is

$n_{\mathrm{kn}}\left(n_{a}\right)=\left(\begin{array}{c}n_{a} \\ 2\end{array}\right)-\left(n_{a}-1\right)=\frac{\left(n_{a}-1\right)\left(n_{a}-2\right)}{2}$.

The underlying relationship between the baselines and our new observables is non-trivial, but will be assumed to hold for any non-redundant array. For redundant arrays this number decreases. We call "complete" a nuller that provides the abovementioned maximum number $n_{\mathrm{kn}}$ of independent observables. The number of independent nulls in the full set is $n_{\text {indep. }}=2 \times n_{k n}$. The results obtained empirically for up to seven inputs are shown in Table 1, along with their expected progression for larger numbers of inputs.

As seen in Eq. (22), an increase in the number of nulled rows decreases the normalization coefficients $a_{i}$ as, in practice, fewer splittings of the input light are necessary to obtain fewer combinations. Our goal may therefore be to construct complete combiners using the minimum number of nulled combinations from the full matrix $\mathbf{M}$, with the intent of increasing its throughput. Expecting this number to be twice the number of kernel nulls (if we consider only pairwise kernel nulls), this produces a very large number $n_{\text {crops }}\left(n_{a}\right)$ of possible combinations:

$n_{\text {crops }}\left(n_{a}\right)=\left(\begin{array}{c}\left(n_{a}-1\right) ! \\ \left(n_{a}-1\right)\left(n_{a}-2\right)\end{array}\right)$.

Only for the cases of three and four inputs is the solution unique $\left(n_{\text {crops }}\left(n_{a}\right)=1\right)$, and all null rows must be kept. For more inputs, this number grows rapidly. Although a large fraction of them are complete, fewer satisfy the conditions detailed in Appendix A for conservation of energy. The following characteristics are shared by the three- and four-input combiners, as well as all the lossless realizations of the cropped five-input combiner:

- All nulls appear in conjugate (or enantiomorph) pairs, which implies that robust observables can be constructed by subtraction.

- Each phasor appears in each column the same number of times (except for that of phase zero which serves as the reference). Equation (23) implies that for a combiner producing $n_{o}=2 n_{\mathrm{kn}}$ nulls, each phasor is used $\left(n_{a}-2\right)$ times for each input.

Enforcing these characteristics as rules for reducing the number of outputs has helped us to identify lossless realizations of the cropped six-input combiner outlined in Sect. 5.2 by reducing the parameter space. The process leading to a valid solution remains one of trial and error: chosen randomly among all the possible arrangements that respect the above-mentioned characteristics, a solution is only accepted when the corresponding combiner is both lossless (see Appendix A) and produces a complete set of kernel nulls.
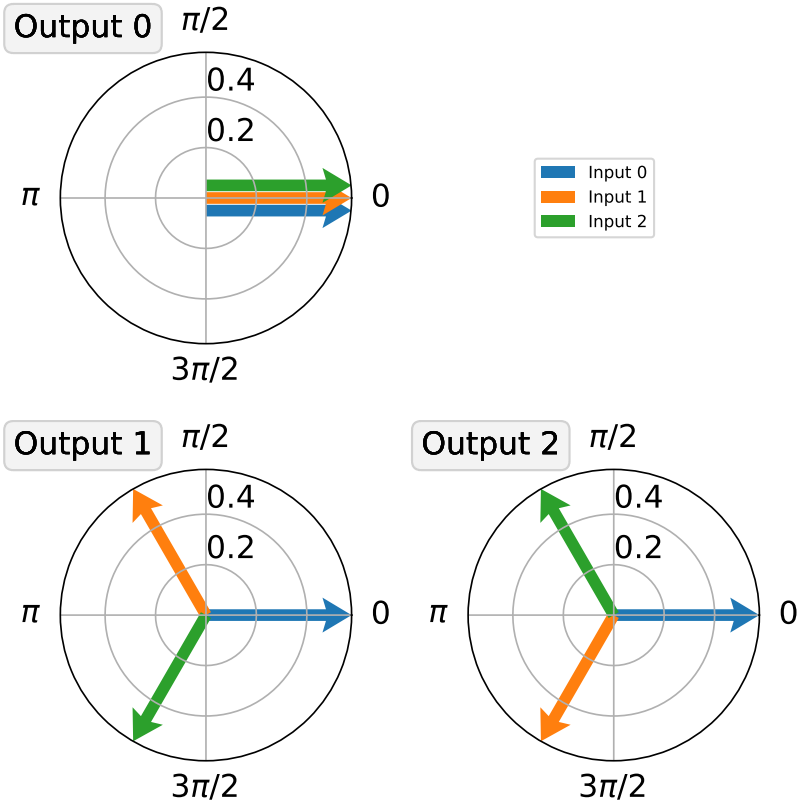

Fig. 4. CMP for a three-input kernel nuller of Eq. (25). The first row corresponds to the bright channel with the overlapping phasors staggered for readability. The two nulled outputs are complex conjugates of one another and form a kernel null.

\section{Examples of combiners}

\subsection{Three-input kernel nuller}

The simplest practical example of this architecture appears for the combination of three inputs. Here the algorithm results in the formation of two enantiomorph nulled outputs. These two outputs will, by subtraction, produce one robust observable. The resulting combiner matrix writes

$\mathbf{M}_{3}=\frac{1}{\sqrt{3}}\left[\begin{array}{ccc}1 & 1 & 1 \\ 1 & e^{\frac{2 j \pi}{3}} & e^{\frac{4 j \pi}{3}} \\ 1 & e^{\frac{4 j \pi}{3}} & e^{\frac{2 j \pi}{3}}\end{array}\right]$.

The combinations offered by this matrix are illustrated in Fig. 4.

As an example, we built a response map of the robust observable produced by this combiner fed by three of the VLTI (von der Lühe 1997) unit telescopes (UTs) observing at zenith. Figure 5 shows the values of the kernel-null observable represented as a two-dimensional function of the relative position of a source normalized by the flux of one aperture. While simpler than that provided in Fig. 7 of Martinache \& Ireland (2018) for the four-input combiner, this pattern retains the same antisymmetric property.

Assuming the practical implementation of the combiner itself can be manufactured either with bulk or integrated optics, this configuration would allow the production of robust high-contrast observables with the least amount of infrastructure. Drawing a parallel between this type of combinations and closure triangles used for closure phases is tempting but misleading. Here, as the combination must be done optically rather than in post-processing, kernel nulling does not scale in the same way. The advantages and drawbacks of using these simple combiners as building blocks is briefly discussed in Sect. 6.1. 


\subsection{Six-input kernel nuller}

We also outline a solution for a kernel-nulling recombiner for six telescopes that could, for example, be used at the focus of the CHARA array. The initial algorithm produces a combiner matrix $\mathbf{M}_{6}$ with 121 rows with redundancy in the off-axis response. It is cropped to $\mathbf{M}_{6}^{\prime}$ using the guidelines offered in Sect. 4.2 to reduce it to the minimum of 21 rows, while making sure the number of independent kernel nulls $n_{\mathrm{kn}}$ is preserved. Furthermore, by enforcing the properties outlined in Appendix A, we make sure that $\mathbf{M}_{6}^{\prime}$ remains the matrix of a lossless beam combiner.

The matrix describing this six-input combiner writes

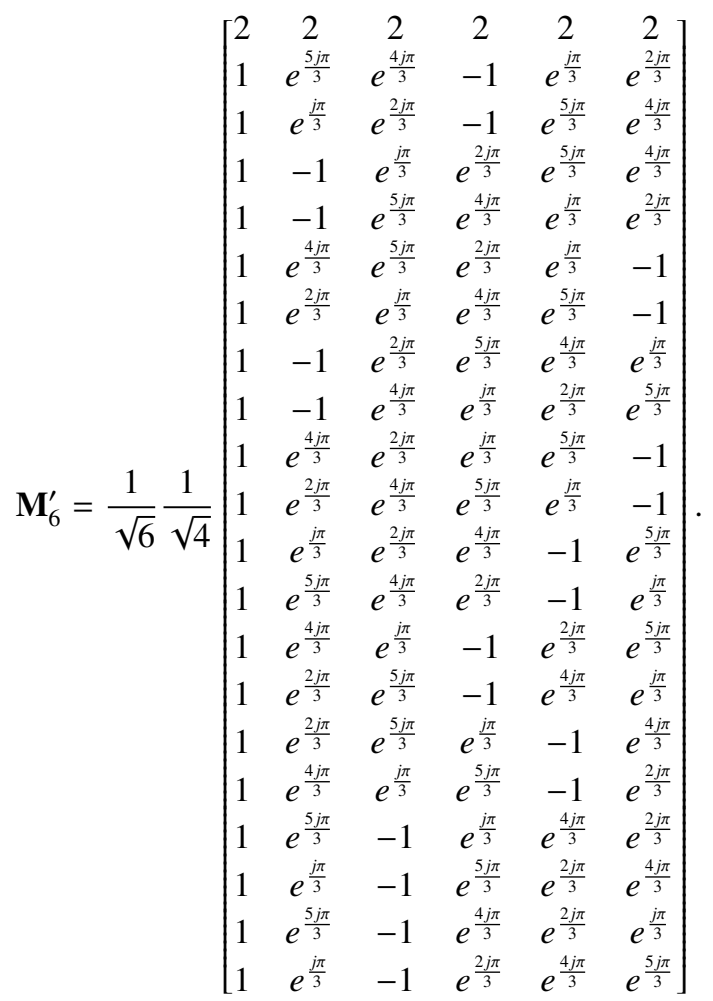

This combiner offers a total of 20 independent nulls, and 10 independent kernel nulls. The corresponding CMP is shown in Fig. 6 and highlights how each aperture contributes to all of the outputs.

To illustrate the astrophysical information gathered by the larger number of kernel nulls, we construct response maps of the kernel-null observables. The plots are shown in Fig. 7 and display the response of each of the observables for the combiner being fed by the CHARA array observing a target at zenith in the $3.6 \mu \mathrm{m}$ wavelength. The patterns reflects the richness of the uv coverage provided by an array like CHARA, and the fact that each output uses information collected by every telescope. As a consequence, each map covers the field of view differently, and brings a new constraint on the properties of the astrophysical scene observed.

\section{Discussion}

No active long-baseline optical interferometer currently provides more than six sub-apertures. However, the masking of monolithic apertures to produce interferometric arrays is an established practice (Tuthill et al. 2010; Jovanovic et al. 2012) that may be used in conjunction with nulling interferometry (Norris et al. 2020). Therefore, the use of even larger combiners may prove to be a viable alternatives to small inner working angle
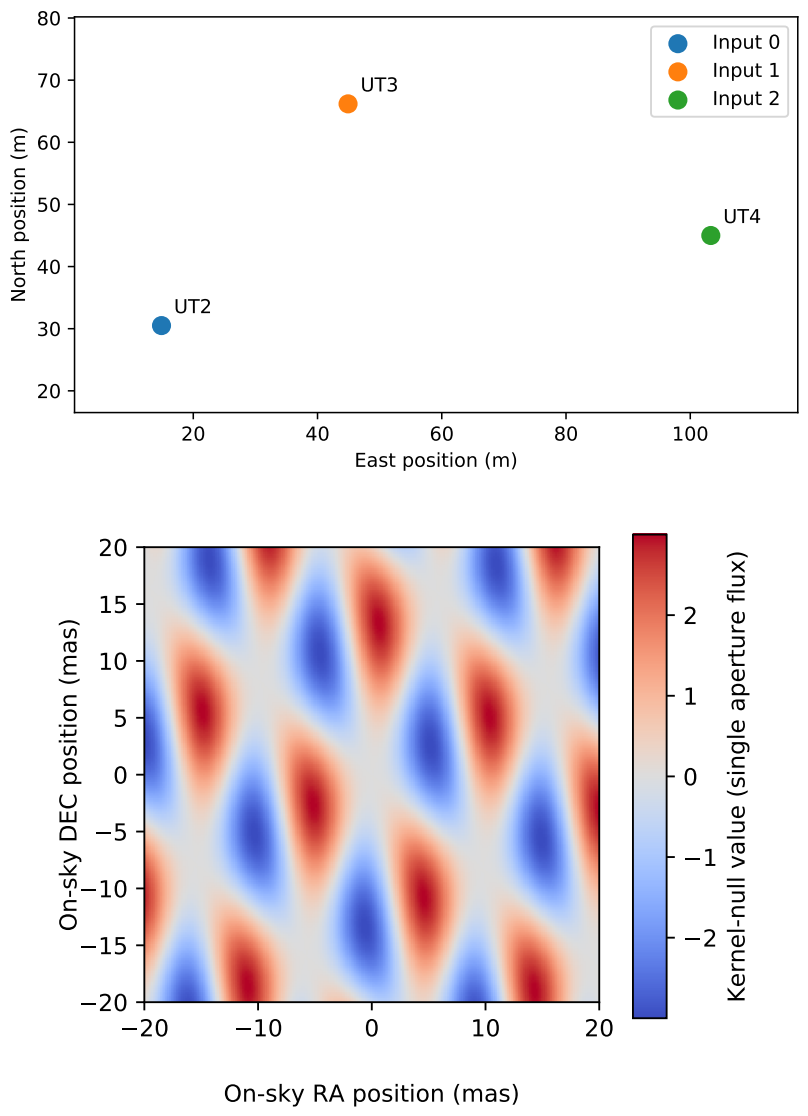

Fig. 5. Top: three telescope configurations picked for the example and corresponding to the positions of three of the VLTI UTs. Bottom: value of the kernel null as a function of the relative position of a source of unit contrast at $3.6 \mu \mathrm{m}$, normalized to the throughput of one aperture. The map is relevant for a target observed at Zenith and would evolve with the projected aperture map. The nature of the response is antisymmetric, as demonstrated in Sect. 3.3.

coronagraphs (Guyon et al. 2006). Their robustness to small aberrations might provide unprecedented contrast performance in the $1-3 \lambda / D$ regime in the near-infrared.

\subsection{Multiplexing nullers}

Instead of building an all-in-one combiner, which may be difficult to construct for a large number of apertures, an alternative approach would be to multiplex several independent nullers, each of which recombines a smaller number of apertures. For example, instead of a six-input nuller producing 20 nulled outputs, one conservative option would be to use two three-input kernel nullers (identical to the one presented in Sect. 5.1) side by side, producing four nulled outputs.

While the second of these two options results in a reassuring higher throughput per output, it can only produce distinct robust observables, whereas the $\mathbf{M}_{6}^{\prime}$ combiners offer ten. Moreover, this multiplexed option also results in two bright channels where some of the off-axis light is also lost, further reducing the overall efficiency of the combiner. In between these two extreme scenarios intermediate solutions can be imagined to alleviate some of their risks and deficiencies, with a modular design multiplexing the nullers much like a number of ABCD combiners are multiplexed inside the beam combiner of VLTI/GRAVITY. 


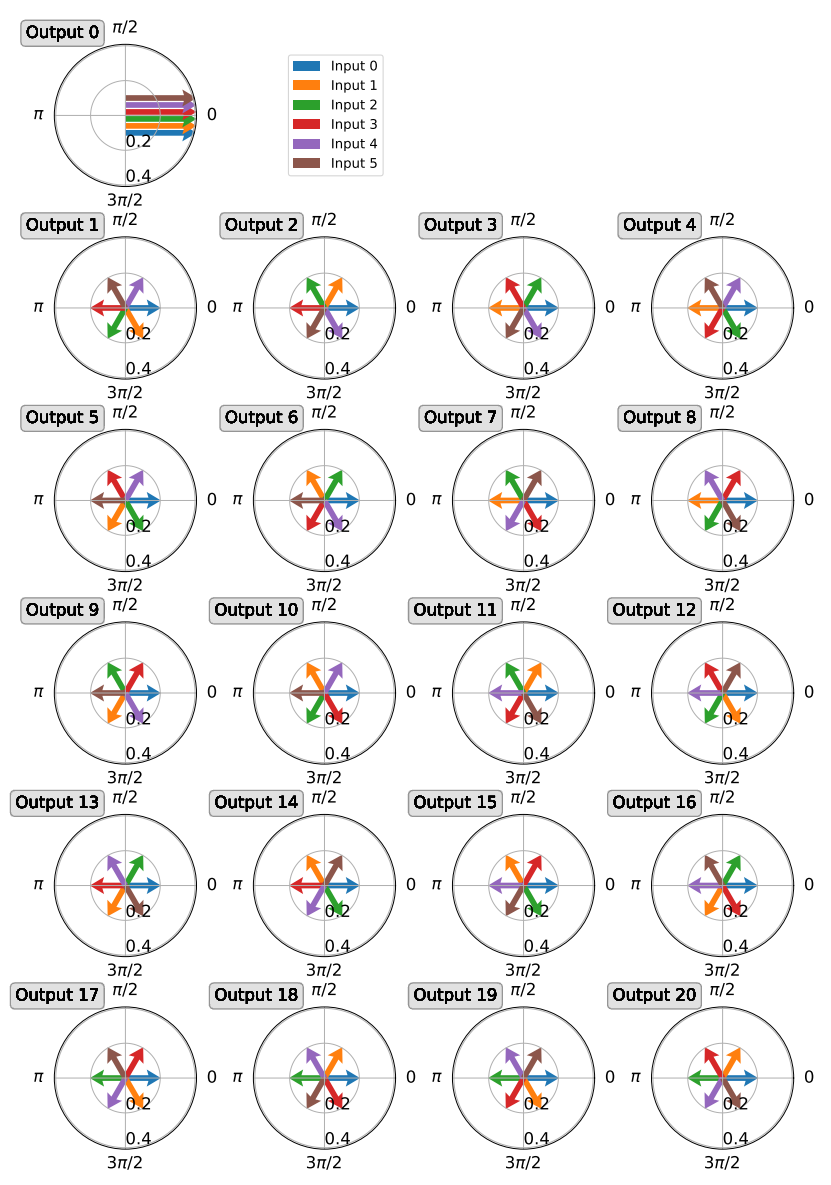

Fig. 6. Representation of the 20 nulled outputs of the six-input beam combiner proposed in Eq. (26). The conjugate pairs that form the ten kernel nulls are represented side by side.

It was already argued by Guyon et al. (2013) that efficient nulling solutions concentrate the most starlight into the smallest number of outputs, which favors the all-in-one combiner over the multiplexed versions. If manufacturability or operational constraints were to prevent the deployment of an all-in-one combiner at the focus of a specific observatory, one way to alleviate this inefficiency could be to recombine the light from the multiple bright outputs into an additional nulling stage so as to extract additional useful observables. This type of architecture, in part inspired by the hierarchical fringe tracker idea of Petrov et al. (2014), might prove a necessary compromise to the implementation of a kernel nuller at the focus of a verylong-baseline observing facility such as the envisioned Planet Formation Imager (Monnier et al. 2016), for which a distributed hierarchical recombination mode seems particularly apt.

\subsection{Evolution of robustness}

In addition to trade-off considerations between the total number of observables and the throughput efficiency of the available options, we must also consider whether the number of inputs has an impact on the phase-noise rejection performance of a kernel nuller.

To evaluate this risk, we trace the evolution of the noise affecting the outputs and their kernels as a function of the amount of phase noise affecting the inputs. We do this for the 3T, 4T, and 6T designs described in the previous sections. For simplicity, this study assumes that the phase noise affecting all
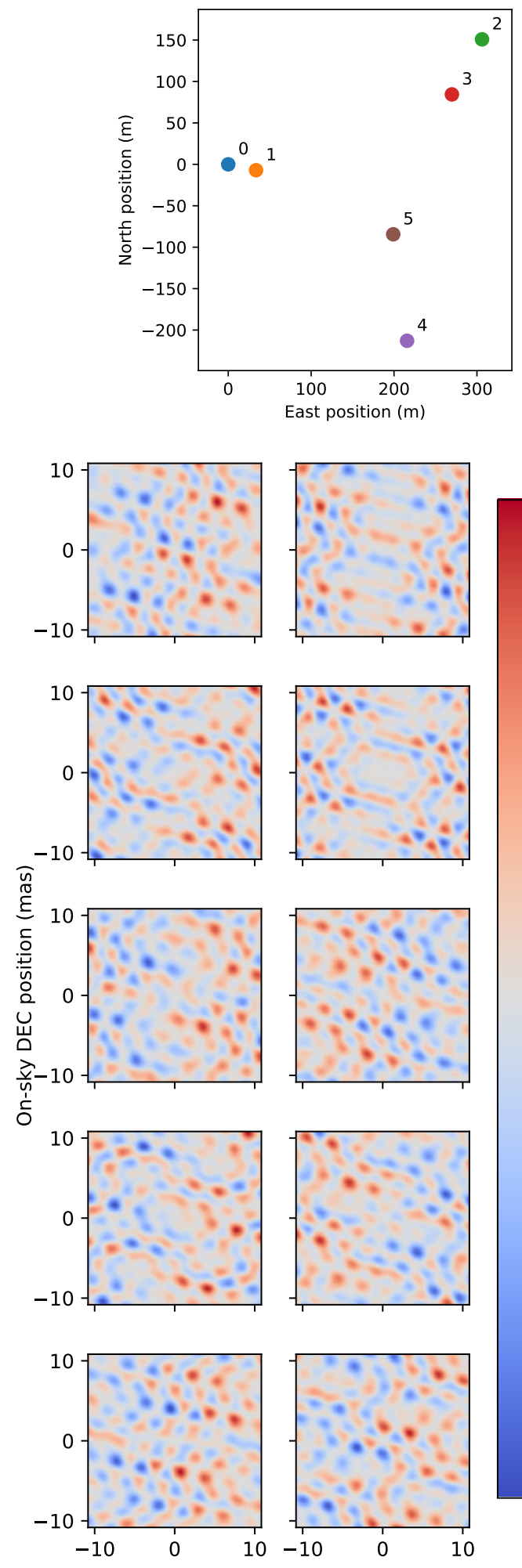

$-0.5$

On-sky RA position (mas)

Fig. 7. Top: six-telescope configuration for the CHARA array used as example. Bottom: value of all ten kernel nulls as a function of the relative position of a source at the wavelength $3.6 \mu \mathrm{m}$ observed at zenith. The transmission is normalized by the flux of a single aperture. Again, each map remains antisymmetric.

inputs is Gaussian, non-correlated, and characterized by a single rms value equally affecting all inputs. Following a Monte Carlo approach, random realizations of input piston errors are 


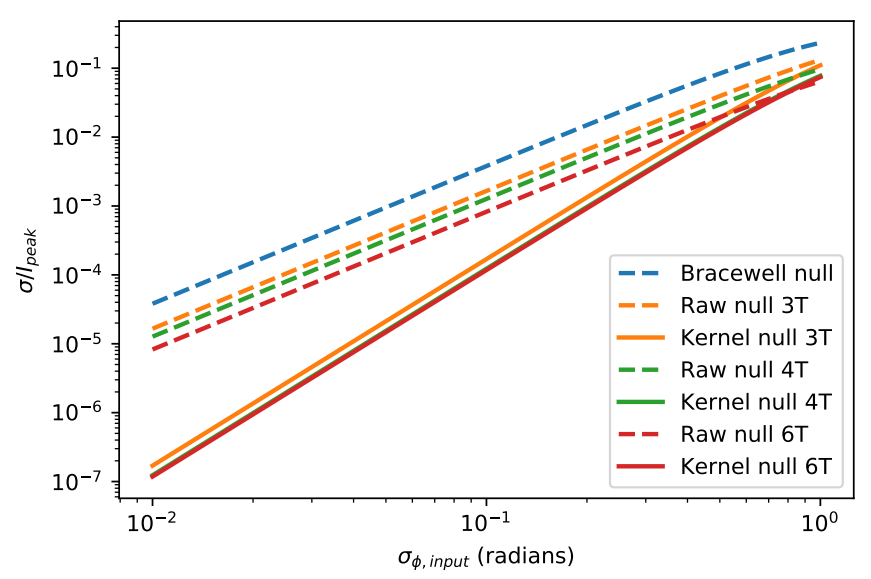

Fig. 8. Propagation of phase noise from the input to the nulled intensities for different kernel-nulling architectures. Values are normalized by the peak transmission of an off-axis signal. The raw nulls (dashed lines) are compared with their corresponding kernel-null observable (solid lines), showing the suppression of second-order phase noise (by a few orders of magnitude) for small input phase error. This effect decreases as the input phase errors depart from the small phase approximation The behavior of the Bracewell nuller is shown for reference (dashed blue lines).

drawn, propagated through the different combiner matrices, and the standard deviation of the output intensities are evaluated. Figure 8 thus shows the evolution of the standard deviation of the output intensities of the nulls and of the kernel nulls of the different architecture normalized by the peak null intensity $I_{\text {peak }}$ from their response map. This therefore constitutes a noise-to-signal ratio of sorts. The simple Bracewell nuller is also added to this study for comparison, as modeled by the combiner matrix:

$\mathbf{M}_{B}=\left[\begin{array}{cc}1 & 1 \\ -j & j\end{array}\right]$.

This plot shows that the larger kernel-nulling combiners provide a rejection of the phase noise that is very similar to the smaller ones, if not slightly better. The improvement on the raw observables may be credited to a manifestation of the central limit theorem affecting the distribution of the sum of a larger number of complex intensities. Further interpretation of this plot must be undertaken with caution. While the distribution of kernel nulls under such conditions is close to Gaussian (Martinache \& Ireland 2018), the distribution of null intensities is not (Hanot et al. 2011) and is therefore poorly described by its standard deviation. While a full performance comparison of the different designs lies outside the scope of the present paper and would include the coupled effects of phase and amplitude fluctuations (Lay 2004), these elements already indicate that kernel nullers recombining a large number of sub-apertures are intrinsically at least as robust to phase noise as their smaller, simpler counterparts. This is an encouraging prospect for single telescope applications of the kernel nuller for which a potentially large number of sub-apertures can be used.

\section{Conclusions}

In this work we offer a new description of the kernel-nuller design introduced by Martinache \& Ireland (2018). This is done by introducing a new graphical representation of the complex matrix that models the nuller and the transformations it operates on the input electric field. Combined with an analytical description of the outputs used to form a kernel, these representations explain the origin of a kernel nuller's main properties: their intrinsic robustness to small input piston and amplitude fluctuations, and their sensitivity to asymmetric features of the observed scene. We show that the same outputs can also be summed so as to fall back on the original outputs of an all-in-one $\mathbf{N}_{4}$ nuller stage; while not robust to perturbations, this can nevertheless provide further astrophysical information.

Our visual and analytical representations help devise a systematic way to build a kernel nuller as a combiner featuring pairs of channels that are enantiomorph in the complex plane. It is this feature that makes two channels equally sensitive to perturbations, although they respond differently to the presence of an offaxis structure. This approach allow us to design kernel nullers for an arbitrary number of apertures, which we apply in this paper to three- and six-aperture arrays.

We discuss the possibility of simplifying kernel nullers that grow in complexity when they recombine a larger number of input beams, for instance using distinct nullers operating in parallel over a subset of input beams. For a given total number of inputs, a global architecture that gives access to a larger number of high-contrast observables is more efficient and offers the means to explore and characterize complex astrophysical scenes. For the same number of inputs, we also note that the total number of outputs for a kernel nuller (exactly twice the number of theoretical closure-phases) is in fact less than that of nonnulling combiners designed to measure the complex visibility of all baselines. Integrated optical circuits in particular already enable the implementation of such complicated designs in small and stable packages, and are a very promising avenue for the construction of these larger kernel-nulling combiners.

While no existing long-baseline optical interferometric facility currently offers the simultaneous combination of more than six apertures, a kernel nuller sampling the pupil of a single telescope could prove to be a valuable complement to a coronagraph, producing high-contrast observations near one resolution element that would be insensitive to the small but ever present adaptive optics residuals. The evaluation of performance in practical implementations including the contribution of coupled phase an amplitude contributions and the consideration of relevant science cases will be the topic of future theoretical and experimental work.

Acknowledgements. We thank Alban Ceau and Coline Lopez for their suggestions to improve the manuscript. KERNEL has received funding from the European Research Council (ERC) under the European Union's Horizon 2020 research and innovation program (grant agreement CoG - 683029).

\section{References}

Angel, J. R. P., \& Woolf, N. J. 1997, ApJ, 475, 373

Boccaletti, A., Chauvin, G., Mouillet, D., et al. 2020, SPHERE+: Imaging Young Jupiters Down to the Snowline, Tech. Rep.

Bracewell, R. N. 1978, Nature, 274, 780

Chauvin, G., Desidera, S., Lagrange, A. M., et al. 2017, A\&A, 605, L9

Colavita, M. M., Serabyn, E., Millan-Gabet, R., et al. 2009, PASP, 121, 1120

Defrère, D., Hinz, P. M., Skemer, A. J., et al. 2015, ApJ, 799

Defrère, D., Hinz, P. M., Mennesson, B., et al. 2016, ApJ, 824, 66

Guyon, O., Pluzhnik, E. A., Kuchner, M. J., Collins, B., \& Ridgway, S. T. 2006, ApJS, 167, 81

Guyon, O., Mennesson, B., Serabyn, E., \& Martin, S. 2013, PASP, 125, 951

Hanot, C., Mennesson, B., Martin, S., et al. 2011, ApJ, 729

Hoffmann, W. F., Hinz, P. M., Defrère, D., et al. 2014, Ground-based and Airborne Instrumentation for Astronomy V, 9147, 914710

Jennison, R. C. 1958, MNRAS, 118, 276

Jovanovic, N., Tuthill, P. G., Norris, B., et al. 2012, MNRAS, 427, 806 
Lacour, S., Tuthill, P., Monnier, J. D., et al. 2014, MNRAS, 439, 4018 Lacour, S., Nowak, M., Wang, J., et al. 2019, A\&A, 623, L11

Lay, O. P. 2004, Appl. Opt., 43, 6100

Loudon, R. 2000, The Quantum Theory of Light, 3rd edn.

Lozi, J., Guyon, O., Jovanovic, N., et al. 2018, Proc. SPIE, 10703, 1070359

Macintosh, B., Graham, J. R., Barman, T., et al. 2015, Science, 350, 64

Marois, C., Macintosh, B., Barman, T. S., et al. 2008, Science, 322, 1348

Martinache, F. 2010, ApJ, 724, 464

Martinache, F., \& Ireland, M. J. 2018, A\&A, 619, A87

Mesa, D., Keppler, M., Cantalloube, F., et al. 2019, A\&A, 632, A25

Monnier, J. D., Ireland, M. J., Kraus, S., et al. 2016, Proc. SPIE, 9907, 990710

Norris, B. R. M., Cvetojevic, N., Lagadec, T., et al. 2020, MNRAS, 491, 4180

Petrov, R. G., Elhalkouj, T., Boskri, A., et al. 2014, Optical and Infrared Interferometry IV, 9146, 91462P

Sauvage, J. F., Fusco, T., \& Petit, C. 2016, J. Astron. Telescopes Instrum. Syst., 2

Schneider, J., Dedieu, C., Le Sidaner, P., Savalle, R., \& Zolotukhin, I. 2011, A\&A, 532, A79

Serabyn, E., Mennesson, B., Martin, S., Liewer, K., \& Kühn, J. 2019, MNRAS, 489, 1291

Tuthill, P., Lacour, S., Amico, P., et al. 2010, Ground-based and Airborne Instrumentation for Astronomy III, 7735, 773510

Velusamy, T., Angel, R. P., Eatchel, A., Tenerelli, D., \& Woolf, N. J. 2003, European Space Agency, Special Publication (ESA SP), 2003, 631

von der Lühe, O. 1997, Science with the VLT Interferometer, ed. F. Paresce (ESO Astrophysics Symposia), 13

Zurlo, A., Vigan, A., Galicher, R., et al. 2016, A\&A, 587, A57

\section{Appendix A: Lossless combiners}

As emphasized by Loudon (2000) in Sect. 3.2, the conservation of energy in a single beamsplitter cube imply that its matrix is unitary. This property can be generalized to larger combiners. The required condition is that the sum of intensities of the inputs is the same as the sum of intensities at the outputs. As the output intensities are gathered in the vector $\mathbf{x}$, this sum also writes

$$
\sum_{i=0}^{n_{\text {outputs }}}\left|x_{i}\right|^{2}=\mathbf{x}^{H} \mathbf{x}
$$

with ${ }^{H}$ designating the Hermitian adjoint (conjugate transpose) operator. Based on Eq. (1) this sum writes

$\mathbf{x}^{H} \mathbf{x}=(\mathbf{M z})^{H} \mathbf{M z}$,

which develops as

$\mathbf{x}^{H} \mathbf{x}=\mathbf{z}^{H} \mathbf{M}^{H} \mathbf{M z}$.

As a consequence we obtain

$\forall \mathbf{z} \in \mathbb{C}^{n_{a}} \sum_{i=0}^{n_{\text {outputs }}}\left|x_{i}\right|^{2}=\sum_{i=0}^{n_{a}}\left|z_{i}\right|^{2} \Longleftrightarrow \mathbf{M}^{H} \mathbf{M}=\mathbf{I}$.

Thus, the following propositions are equivalent:

- $\mathbf{M}$ is the matrix of a lossless beam combiner;

- $\mathbf{M}$ is semi-unitary on the left;

- $\mathbf{M}^{H}$ is the left inverse of $\mathbf{M}$;

- The columns of $\mathbf{M}$ are orthonormal;

- All the singular values of $\mathbf{M}$ are equal to one. 